\title{
Geminga: A Cooling Superfluid Neutron Star
}

\author{
Dany Page \\ Department of Astronomy, Columbia University \\ 538 West 120th Street, New York, NY 10027
}

\begin{abstract}
We compare the recent temperature estimate for Geminga with neutron star cooling models. Because of its age $\left(\sim 3.4 \times 10^{5} \mathrm{yr}\right)$, Geminga is in the photon cooling era. We show that its surface temperature $\left(\sim 5.2 \times 10^{5} \mathrm{~K}\right)$ can be understood by both types of neutrino cooling scenarios, i.e, slow neutrino cooling by the modified Urca process or fast neutrino cooling by the direct Urca process or by some exotic matter, and thus does not allow us to discriminate between these two competing schemes. However, for both types of scenarios, agreement with the observed temperature can only be obtained if baryon pairing is present in most, if not all, of the core of the star. Within the slow neutrino cooling scenario, early neutrino cooling is not sufficient to explain the observed low temperature, and extensive pairing in the core is necessary to reduce the specific heat and increase the cooling rate in the present photon cooling era. Within all the fast neutrino cooling scenarios, pairing is necessary throughout the whole core to control the enormous early neutrino emission which, without pairing suppression, would result in a surface temperature at the present time much lower than observed.

We also comment on the recent temperature estimates for PSR 0656+14 and PSR 1055-52, which pertain to the same photon cooling era. If one assumes that all neutron stars undergo fast neutrino cooling, then these two objects also provide evidence for extensive baryon pairing in their core, but observational uncertainties also permit a more conservative interpretation, with slow neutrino emission and no pairing at all. We argue though that observational evidence for the slow neutrino cooling model (the "standard" model) is in fact very dim and that the interpretation of the surface temperature of all neutron stars could be done with a reasonable theoretical a priori within the fast neutrino cooling scenarios only. In this case, Geminga, PSR 0656+14, and PSR 1055-52 all show evidence of baryon pairing down to their very centers.

The Astrophysical Journal, in press.
\end{abstract}

Subject headings: dense matter $\quad-$ stars: neutron - stars: x-rays 


\section{INTRODUCTION}

The study of the thermal evolution of young neutron stars offers the possibility of obtaining unique information about the structure of compressed cold nuclear matter. Early cooling after the supernova explosion is driven by neutrino emission, whose rate is a very sensitive function of the state of that matter. Moreover, both the neutrino cooling and the later photon cooling are strongly affected by the occurence of pairing ( $\grave{a}$ la BCS) of the baryonic components of the star's core. These two aspects combined result in a wide range of predicted surface temperatures and give us two handles by which to extract information about the composition and pairing state of dense matter through comparison of neutron star cooling calculations with observations of neutron stars of known ages.

Observational candidates for this purpose must have an age well below $10^{6} \mathrm{yr}$ since, after this, the star has exhausted its initial heat content and its (much lower) temperature depends on other mechanisms. Interstellar absorption is significant at the photon energies corresponding to the expected surface temperatures, of the order of $10^{6} \mathrm{~K}$ or lower, and hence the star must be not too far away from us and must be in a region of low interstellar absorption. Until recently, only three neutron stars fullfilled these two criteria of relative youth and closeness, all three located within the local bubble of low interstellar matter density surrounding the Sun: PSR 0833-45 (Vela), PSR 0656+14, and PSR 1055-52. They provided the only reliable data to compare with theoretical models. Some other objects, for example PSR 0531+21 (Crab; Harnden \& Seward 1984) or the neutron star in the supernova remnant 3C58 (Becker, Helfand \& Szymkowiak 1982), located at farther distances have only given rough upper limits of their surface temperatures. We refer to Ögelman (1991) for a review of the pre-ROSAT observational situation.

With the recent demonstration by Halpern \& Holt (1992) that Geminga is a neutron star, a new candidate is now available. The quality of the ROSAT data makes this one of the best cases of detection of thermal radiation from a neutron star surface to date, and the data analysis (Halpern \& Ruderman 1993) is the most detailed performed so far. Geminga is one of the closest neutron stars and is located within the local interstellar bubble; Gehrels \& Chen (1993) recently argued that the Geminga supernova may have actually been the cause of this bubble.

Comparison of Geminga's age and temperature with published models of neutron star cooling shows that the data can be accomodated by a variety of models. Several of the direct Urca cooling scenarios of Page \& Applegate (1992), the kaon condensate cooling scenario of Page \& Baron (1990), and some of the pion condensate cooling scenarios of Umeda et al. (1992) work, while none of the fast cooling models, either with quarks or pion 
condensate, of Van Riper (1991) is successful. Some, but not all, of the "standard" cooling models presented by Nomoto \& Tsuruta (1986, 1987), Shibazaki \& Lamb (1989), Page \& Baron (1990), Van Riper (1991), Page \& Applegate (1992), and Umeda et al. (1992, 1993) are also successful. In this paper, we will look at the various ingredients of these models and determine which ones are crucial for compatibility with this new neutron star. A preliminary version of this work has been presented in Page (1992).

The pulsars PSR 0656+14 and PSR 1055-52 have ages similar to Geminga's and fit into the study of the present work. Since the analyses of the ROSAT observations of these two objects have been published recently (Finley, Ögelman \& Kiziloğlu 1992; Ögelman \& Finley 1993) we will also discuss them briefly.

The structure of the paper is as follow. Section 2 presents the observational data on Geminga. Section 3 describes the general physics of neutron star cooling relevent to our present purpose. Section 4 discusses the various fast neutrino cooling scenarios, and section 5 presents detailed calculations within the slow neutrino cooling scenario. Comparison with Geminga is done in sections 4 and 5 while section 6 comments on the relevence of the previous results for other pulsars, in particular for PSR 0656+14 and PSR 1055-52. Section 7 contains our conclusions.

\section{GEMINGA}

\subsection{Geminga's Age}

The Geminga period as measured by Halpern \& Holt (1992) is $P=0.2370974 s \pm 0.1 \mu s$. The earlier observation by $C O S-B$ and the later ones by $G R O$ have slightly different $P$ s which lie very accurately on a straight line (Bignami \& Caraveo 1992) and give a practically constant period derivative of $\dot{P}=1.099 \pm 0.001 \times 10^{-14} s s^{-1}$ over a span of $16 \mathrm{yr}$. The corresponding spin-down age is $\tau=P / 2 \dot{P}=3.4 \times 10^{5} \mathrm{yr}$. This age is obtained with a braking index $n=3$, i.e., with magnetic dipole braking. We will consider a range of ages corresponding to braking indices $n=2$ and 4 ,

$$
2.3 \times 10^{5} \mathrm{yr} \leq t \leq 6.8 \times 10^{5} \mathrm{yr}
$$

the upper value probably being an overestimate. We refer to Michel (1991) and Lyne \& Graham-Smith (1990) for discussions of the reliability of the spin-down age as an indicator of the true age. 


\subsection{Geminga's Temperature}

In the first estimate of the star's temperature, Halpern \& Holt (1992) fitted the two apparent components of the spectrum with a blackbody and a power law. The best fit gave a blackbody temperature of $T=3-4 \times 10^{5} \mathrm{~K}$. In a second, more detailed analysis Halpern \& Ruderman (1993) replaced the power-law component by a second blackbody with higher temperature and argued that this hotter component $\left(T \cong 3 \times 10^{6} \mathrm{~K}\right)$ is due to emission from a reheated polar cap (this temperature is too high to be explained only by the anisotropy of heat transport in the crust in presence of a magnetic field). They obtained for the main surface emission a temperature $T=5.2 \pm 1.0 \times 10^{5} \mathrm{~K}$. From the luminosities of these two components, they concluded that the ratio of the areas of the hot and cold emitting regions is about $3 \times 10^{-5}$. Moreover, it is likely that the surface is not emitting uniformly and that some colder region is not being seen, making the concept of "surface temperature" an ambiguous one. However, cooling calculations give as an output the effective temperature, i.e., a total luminosity, and the presence of a cooler region would reduce the luminosity and make the effective temperature somewhat lower than the inferred value of $5.2 \pm 1.0 \times 10^{5} \mathrm{~K}$.

All these analyses use blackbody spectra, but the surface of a neutron star cannot be expected to be a perfect blackbody. Romani (1987) has calculated more realistic spectra for various surface chemical compositions without magnetic field. Miller (1992) and Shibanov et al. (1992) have partially extended these results by including the magnetic field effects. The general trend of these results, for $\mathrm{H}$ or He atmospheres, is that there is an excess emission in the Wien tail of the spectrum, compared to a blackbody, and the excess falls within the Einstein and ROSAT detector ranges. This excess is reduced if metals are present (because of absorption edges) or when the effects of the magnetic field are taken into account. As a consequence, the use of these spectra would lower the measured temperature. Finally, some contamination from a surrounding nebula and/or some surface reheating by gamma rays or

particles from the magnetosphere cannot be excluded. See Halpern \& Ruderman (1993) for a discussion.

We will take for comparison with our calculations an effective temperature of

$$
4 \times 10^{5} \mathrm{~K} \leq T_{e} \leq 6 \times 10^{5} \mathrm{~K}
$$

but insist that it must be taken as an upper limit for the above mentioned reasons. Being determined from the spectrum, this temperature is independent of the distance, mass, and radius of the star and is the "temperature at infinity" $T^{\infty}$, i.e., the redshifted temperature. 


\section{THE PHYSICS OF NEUTRON STAR COOLING}

At an age of a few multiples of $10^{5} \mathrm{yr}$ Geminga is well into the isothermal phase (Nomoto \& Tsuruta 1987); its internal temperature $T_{i}$ is uniform except for a gradient in the envelope just below the surface. The thermal evolution of the star is determined by energy conservation,

$$
\frac{d E}{d t}=C_{v} \frac{d T_{i}}{d t}=-L_{\nu}-L_{\gamma}
$$

where $E$ is the total thermal energy of the star, $C_{v}$ its total specific heat, and $L_{\nu}$ and $L_{\gamma}$ the total neutrino and photon luminosities (general relativistic correction factors are omitted here but were included in our calculations). The photon luminosity is $L_{\gamma}=4 \pi R^{2} \sigma T_{e}^{4} \propto T_{i}^{2.2}$, where the effective temperature $T_{e}$ is converted into an internal temperature $T_{i}$ according to the $T_{i}-T_{e}$ relationship calculated by Gudmundsson, Pethick \& Epstein $(1982,1983)$. The neutrino emissivities are proportional to $T_{i}^{6}$ or $T_{i}^{8}$ (see Table 11), hence photon emission from the surface will eventually dominate over neutrino emission when the temperature has dropped sufficiently. This turnover happens before the star reaches $10^{5} \mathrm{yr}$ : Geminga is thus well into the photon cooling era. In this phase the cooling rate is mostly determined by the total specific heat of the star, but the actual surface temperature also depends on the

previous neutrino cooling, which can be considered as giving the initial condition for photon cooling. Our cooling curves are calculated with the Heyney-type code already presented in Page \& Baron (1990) and Page \& Applegate (1992) which solves the general relativistic heat transport and energy balance equations. The reader is refered to these papers for more details.

\subsection{Neutrino Processes}

The dominant neutrino emission processes occur in the core of the star and are variants of beta and inverse beta decay. Table 1 shows the approximate emissivities of several processes for comparison. One can divide them into slow and fast neutrino emission, according to wether they involve four or two baryons. The large difference between slow and fast processes comes mainly from phasespace considerations: Fermi's "Golden rule" tells us that the rate is proportional to the total phasespace volume available for both initial and final particles, and for fermions this volume is proportional to $k_{B} T / E_{F}$ where $E_{F}$ is the particle Fermi energy. Typical nucleon Fermi energies in neutron star cores are of the order of a few tens to a few hundreds of $\mathrm{MeV}$; if one takes a typical temperature 
of $T=10^{9} \mathrm{~K} \cong 0.1 \mathrm{MeV} / k_{B}$ and $E_{F} \sim 10^{2} \mathrm{MeV}$ then a four fermion process is about $\left(k_{B} T / E_{F}\right)^{2} \sim 10^{-6}$ time weaker than a two fermion process, which is what the direct Urca - modified Urca emissivities show (see Table 1). Participation of a meson (pion or kaon) in a process does not introduce any phase space limitation since these are bosons, but strong interactions effects, and strangeness violation in the case of kaons, reduce the efficiency of meson processes compared to the simple direct Urca process. Hyperons may also constitute a large fraction of the core baryons (see, e.g., Glendenning 1985) and will then participate into either modified (Maxwell 1987) or direct (Prakash et al. 1992) Urca processes, depending on their relative concentrations, but with somewhat lower emissivities compared to the corresponding nucleon processes. We refer to Pethick (1992) and Prakash (1993) for recent reviews of the neutrino emission problem. While there is still doubt about which fast process can actually occur, the number of presently proposed channels is so large that it is becoming difficult to believe that none of them is permitted and that neutron star cooling follows the old "standard" model with only the slow modified Urca process. Nevertheless, awaiting a conclusive argument on this point, we will still consider both the fast and slow cooling scenarios.

Deconfined quarks may be present in the center of massive neutron stars and are also copious neutrino emitters. They thus belong to the fast neutrino cooling scenario but obviously require a separate treatment. We will not consider them explicitly here.

\subsection{The Boundary Condition}

An important ingredient is the above mentioned $T_{i}-T_{e}$ relationship. Gudmundsson et al. $(1982,1983)$ were the first to present a detailed study of it but did not include the effect of the magnetic field $\vec{H}$ which enhances the heat transport parallel to $\vec{H}$ and strongly suppresses it in the perpendicular direction. The extensive magnetic envelope calculations of Hernquist (1985) and Van Riper (1988) for transport parallel to $\vec{H}$ show that for a given inner temperature $T_{i}$ the surface temperature $T_{s}$ is raised, compared to the nonmagnetic case, but by no more than $50 \%$ even with a field of $10^{14} \mathrm{G}$ (the surface magnetic field of Geminga is estimated to be about $1.6 \times 10^{12} \mathrm{G}$ ). When the field is at an angle to the surface, $T_{s}$ should be lower, and by simple geometric considerations Hernquist (1985) argued that the magnetic effects when including a global field configuration are probably very small. Schaaf (1990a,b) has performed envelope calculations with an arbitrary orientation of $\vec{H}$ with respect to the surface, and his results can be used to estimate the surface temperature distributions $T_{s}(\theta, \phi)$ resulting from various magnetic field configurations 
and the corresponding effective temperatures $T_{e}$. Preliminary results (Page 1994) confirm Hernquist's point that the $T_{i}-T_{e}$ relationship depends only weakly on the magnetic field. We will consequently use the zero-field relationship here, which should introduce an error of no more than a few percent. 


\subsection{The High-Density Equation of State}

The equation of state (EOS) has two functions in our models, the first being simply to give the global structure of the star, i.e., the density versus radius profile as a solution of the Oppenheimer-Volkoff equation of hydrostatic equilibrium, and the second being to determine the chemical composition. Obviously, the slow and fast neutrino cooling cases are associated with quite different EOSs at supranuclear density, and we will discuss them separately.

For modeling the slow neutrino cooling, we consider five different EOSs from modern calculations: one relativistic, MPA (Müther, Prakash \& Ainsworth 1987), two nonrelativistic, FP (Friedman \& Pandharipande 1981) and WFF(av14) (Wiringa, Fiks \& Fabrocini 1988), and two parametric, PAL32 and PAL33 (together PAL: Prakash, Ainsworth \& Lattimer 1988), whose properties are intermediate to the above three. Each one of these calculations gives the energy per baryon for neutron matter and symmetric nuclear matter, from which we obtain the EOS for matter in $\beta$-equilibrium and the chemical composition following Wiringa et al. (1988), or Prakash et al. (1988) for PAL. The properties of these EOSs related to neutron stars are summarized in Table 2; they encompass a broad range of stiffness with maximum masses of $1.68 M_{\odot}-2.44 M_{\odot}$, proton fractions in a $1.4 M_{\odot}$ star of $2.0 \%-11.4 \%$ and radii of a $1.4 M_{\odot}$ star of $10.6 \mathrm{~km}-12.5 \mathrm{~km}$. We have rejected EOSs

with proton fractions large enough to allow the direct Urca process in a $1.4 M_{\odot}$ star, but do consider MPA, PAL32, and PAL33 which allow it at higher masses (notice that the PAL32 EOS gives a $1.4 M_{\odot}$ star on the verge of allowing the direct Urca process). Two EOSs, BPS (as listed in Baym, Pethick \& Sutherland 1971) and PS (Pandharipande, Pines \& Smith 1976) have been very popular in neutron star cooling studies. We do not use the BPS EOS for modeling the slow cooling since this extremely soft EOS (which gives a density above 10 times nuclear matter density in the center of a $1.4 M_{\odot}$ star) is actually built on a high-density EOS with hyperons (Pandarhipande 1971) in which the hyperonic direct Urca is allowed and this EOS thus belongs to the fast neutrino cooling case (hyperons appear at a star mass of $0.4 M_{\odot}$ in this model). The softening of this EOS is due in an essential way to the presence of the hyperons, and nothing similar can be expected with only nucleons. The PS EOS has already been considered in previous works (Nomoto \& Tsuruta 1986, 1987; Van Riper 1991), and we will simply quote their results below. However, in this extremely stiff EOS the neutrons form a three-dimensional lattice and thus have a totally different specific heat and a different neutrino emissivity than liquid neutrons, two facts not taken into account in the models, and the stiffness of this EOS is inseparable from to the lattice structure.

The choice of the EOS for fast neutrino cooling models is not as important as for slow 
cooling at the present stage of development of the theory. There are several other factors which have much more influence, for example the critical density at which fast neutrino emission turns on, the fast neutrino emission which is actually at work, the occurence of baryon pairing, etc., all of which are at best poorly known. Therefore, calculations of fast cooling have often been done with various EOSs in a partially justified, careless way. However, one model has been developed with much detail, based on the ALS model of dense matter (Takatsuka et al. 1978), which is somewhat inspired by the PS EOS, but where the lattice structure is one-dimensional and where two-dimensional nucleon pairing occurs in planes orthogonal to the lattice direction (see Tamagaki 1992 for a general description). In this model, charged pion condensation develops at high density, but the resulting EOS is close to the FP EOS at low density below the condensation threshold, i.e., the stiffness of the PS EOS has disappeared. Cooling calculations within the ALS model have been performed by Umeda et al. (1992), and their results will be used below.

\subsection{Nucleon Pairing}

Pairing à la BCS (superfluidity) of the nucleons in the neutron star core has a dramatic effect on the cooling because it suppresses both the neutrino emission and the specific heat. The pairing is in the ${ }^{1} S_{0}$ partial wave at low density and then shifts to the ${ }^{3} P_{2}$ partial wave at higher density. The protons in the core and the neutrons in the inner crust are expected to be paired in the ${ }^{1} S_{0}$ partial wave while the neutron pairing shifts to the ${ }^{3} P_{2}$ partial wave in the core. The pairing of protons in the ${ }^{3} P_{2}$ partial wave seems never to have been considered, probably on the grounds that its critical temperature would be very low. At still higher densities, the pairing should shift to the ${ }^{1} D_{2}$ partial wave (the next partial wave in which the free nucleon-nucleon interaction is attractive), but the estimated corresponding critical temperature is too low for this type of pairing to be of any interest (Amundsen \& Østgaard 1985b). Theoretical calculations of $T_{c}$ are extremely difficult and the presently published values are still highly uncertain except in the case of crust neutron ${ }^{1} S_{0}$ pairing, where reasonable agreement has been obtained between the various latest calculations. Figure 1 shows most of the presently published calculations of critical temperatures for core neutron and proton pairings. One sees that both the maximum value of $T_{c}$ and the density range where it is nonzero are very uncertain, particularly in the neutron case. For the ${ }^{1} S_{0}$ proton pairing, the latest calculation (Wambach, Ainsworth \& Pines 1991) was the first to include in a consistent way the neutron background and shows a strong reduction of $T_{c}$, but with a density dependence apparently different from that found in earlier calculations. However, this result depends strongly on the relative densities of neutrons and protons, 
and thus, calculations with a different proton fraction (a poorly known quantity) may give quite different results (Ainsworth 1992). Medium dispersion effects (i.e., change in the nucleon effective mass) have an enormous effect, as shown in Figure 1 b by the differences between the curves $\mathrm{AO}$ and $\mathrm{T} 72$, where the effective mass $m^{*} \equiv M^{*} / M$ is obtained in a self-consistent way and the corresponding curves labeled with $m^{*}=1$ for which the effective mass is forced to the free mass value. (The Hoffberg et al. 1970 calculation used $m^{*}=1$.) Of course, $m^{*}$ is also poorly known at high density. Further extensive calculations within the ALS model (Takatsuka \& Tamagaki 1982, and references therein) with and without pion condensation give values of $T_{c}$ for ${ }^{3} P_{2}$ neutron pairing that are between the two extremes shown in Figure $\mathbb{1 b}$ as T72 and T72 $\left(m^{*}=1\right)$. For ${ }^{3} P_{2}$ neutron pairing, background effects beyond the first order ones considered in the results shown in Figure 1 $1 \mathrm{~b}$ and similar to the effects considered by Wambach et al. (1991) for ${ }^{1} S_{0}$ proton pairing have been studied (Jackson et al. 1982). The results indicate that, in this case, background effects strongly enhance the pairing; thus, values of $T_{c}$ higher than shown in Figure $1 \mathrm{~b}$ and extending to higher densities are quite possible. In light of this, the case for proton ${ }^{3} P_{2}$ pairing should also be considered seriously, as should ${ }^{1} D_{2}$ pairing. In other words, we know neither the value of $T_{c}$ in the core nor the relevent density range, neither for neutrons nor for protons, but it is reasonable to expect large values extending to high densities. If hyperons are present, one can expect that they will also pair for the same reasons nucleons do. With regard to quarks, pairing is also very probable (Bailin \& Love 1984).

The effect of pairing is to reduce the phase space available for excitations. As a result, both the specific heat of the paired component and the neutrino processes in which it participates will be suppressed. We treat this effect on $C_{v}$ following Levenfish \& Yakovlev (1993; see also Gnedin \& Yakovlev 1993), who performed detailed calculations of the reduction factors in cases both of isotropic ${ }^{1} S_{0}$ and anisotropic ${ }^{3} P_{2}$ pairing. For the neutrino emissivity suppression, we simply use a Boltzmann factor $\exp (-\Delta / k T)$, where $\Delta$ is the pairing gap, which is not very accurate but has no serious consequence here since we consider only the photon cooling era.

\section{FAST NEUTRINO COOLING SCENARIOS}

Fast neutrino cooling encompasses a variety of different scenarios, kaon or pion condensate, direct Urcas with nucleons and/or hyperons and/or isobars, quark matter, and so forth which share the characteristic that their neutrino emissivities are many orders of magnitude higher than the modified Urca process emissivity. As a result, whenever one of 
these processes is allowed to operate freely, the resulting surface temperature, once the star has reached isothermality, is far below any of the presently available observational estimates (Page \& Baron 1990; Van Riper 1991; Page \& Applegate 1992; Page 1992; Umeda et al. 1992), including the new Geminga observation. This raw picture is strongly altered if the neutrino-emitting baryons (nucleons, hyperons, quarks, etc.) become paired. As presented by Page (1989) for the kaon condensate case and by Page \& Applegate (1992) for the direct Urca with nucleons case, the early suppression of the neutrino emission due to the pairing gap can keep the surface temperature $T_{s}$, after isothermalization and until an age of about $10^{5} \mathrm{yr}$, anywhere between approximately $2 \times 10^{5} \mathrm{~K}$ and $1.5 \times 10^{6} \mathrm{~K}$. The actual value of $T_{s}$ is then only a function of the pairing critical temperature $T_{c}$ of the neutrino emitting fluid(s) (to be precise, since $T_{c}$ is density dependent, $T_{s}$ is a function of the lowest value of $T_{c}$ within the "pit" of fast neutrino emission). In a multicomponent system like nucleons + hyperons, various direct Urca processes can operate simultaneously (Prakash et al. 1992), and, for the star not to drop into invisibility, all of them have to be stopped by having one of the participating baryons paired. In particular, if both $\Lambda$ and $\Sigma^{-}$are present together, they undergo a purely hyperonic direct Urca process, and thus, one of them must be paired. One can expect that $T_{c}$ is lower for hyperons than for nucleons if the former have weaker interactions than the latter, and the star's temperature would then be controlled by hyperon pairing.

The models of Page \& Baron (1990), Umeda et al. (1992), and Page \& Applegate (1992) of kaon, pion, and nucleon direct Urca cooling, respectively, with superfluidity suppression easily accomodate the estimated surface temperature of Geminga. The crucial point, however, is that pairing has to occur down to the very center of the star and the critical temperature must be higher than $10^{9} \mathrm{~K}$ everywhere. If even a very small region is left unpaired, it will drive the surface temperature well below the observed value of $5 \times 10^{5}$ K. For example, the $1.4 M_{\odot}$ case of Page \& Applegate (1992), with a direct Urca emitting pit of only $0.038 M_{\odot}$, has a temperature of $1.5 \times 10^{5} \mathrm{~K}$ at Geminga's age if pairing does not occur. Such a low surface temperature would make Geminga's surface practically unobservable by $R O S A T$ (the hot polar cap would of course still be seen).

It is not possible to distinguish between the various neutrino emission processes from this analysis only: both a kaon (or pion) condensate and the direct Urca, even if their emissivities differ by three orders of magnitude, are compatible with the Geminga data when pairing is taken into account, but with different values for $T_{c}$. The $T_{c}$ values needed are within the range of theoretical predictions, but this range is so large it can accomodate almost any data. Moreover, even within one given neutrino emission scheme very different values of $T_{c}$ are possible: for example the models labeled HGRR and 0.1HGRR of Fig. 2 in Page \& Applegate (1992), i.e., direct URCA and ${ }^{3} P_{2}$ neutron pairing with $T_{c}$ from 
Hoffberg et al (1970) or with the same $T_{c}$ multiplied by 0.1 , are both acceptable within the observational uncertainty in the Geminga data.

It should be mentioned that internal heating by friction of the crust neutron superfluid can significantly alter the thermal evolution of a neutron star when its temperature, and thus its specific heat, is low. The models of fast cooling with pions and heating of Umeda et al. (1993), without core nucleon pairing, can produce a star with a surface temperature at Geminga's age of at most $3 \times 10^{5} \mathrm{~K}$ when heating is at its maximum strength (instead of $1.5 \times 10^{5} \mathrm{~K}$ without heating). This is lower that the Geminga temperature considered here (measured with a blackbody spectrum) but may be high enough if Geminga is actually cooler.

\section{THE SLOW NEUTRINO COOLING SCENARIO}

Slow neutrino cooling is a well-defined scenario based on the conservative hypothesis that the neutron star core is made exclusively of neutrons and protons (plus electrons and muons to preserve charge neutrality, but no charged pions, kaons, quarks, etc.) with a proton fraction low enough for the direct Urca process to be forbidden (Lattimer et al. 1991), and its predictions for surface temperatures are much more restrictive than those of the fast neutrino cooling scenarios. We defer a detailed study to later work and only analyze here the photon cooling era relevant to Geminga.

The photon energy loss can be calculated accurately since the core temperature-effective temperature relationship is known quite accurately, even in presence of a magnetic field. We take the core neutrino emissivity of the modified Urca process and the two associated neutral current bremstrahlung processes from Friman \& Maxwell (1979). Of critical importance here is the total specific heat of the star, which depends on the EOS and chemical composition (proton fraction). Pairing of nucleons is essential here because of its suppression of the specific heat. Table 3 shows the contribution to the normal (i.e., without pairing) specific heat of the various components in a $1.4 M_{\odot}$ star at a temperature $T=10^{9} \mathrm{~K}$ for our five EOSs. Pairing will suppress $C_{v}$ exponentially when $T \ll T_{c}$ and the corresponding specific heat will practically disappear. In all our calculations, the crust neutrons are paired using the gap from Ainsworth, Wambach \& Pines (1989) and their contribution to $C_{v}$ is thus strongly reduced; the crust electrons make a negligible contribution as does the crust lattice. One can see from Table 3 that the core neutrons contribute about three-fourths of the total specific heat, the protons one-fourth and the core electrons about $5 \%$, independent of the EOS. 
Because of the large theoretical uncertainty about the actual value of the pairing critical temperature $T_{c}$ for both neutrons and protons in the core as well as the uncertainty on the density dependence of $T_{c}$ we first consider density independent $T_{c}$; i.e., we force pairing in the whole core and take $T_{c}=2 \times 10^{9} \mathrm{~K}$. The resulting cooling curves of a $1.4 M_{\odot}$ star for our five EOSs are shown in Figures $2 \mathrm{a}-2 \mathrm{~d}$ and are compared with the Geminga data. Because in this photon cooling era the determining factor is the total specific heat, the five EOSs give basically identical results since the contribution of the various components to $C_{v}$ is only weakly dependent on the EOS and pairing, when assumed, is present in the whole core. When none of the core nucleons is paired (a) the theoretical results are consistent only with the higher surface temperature $T_{s}$ and the older age: considering that this $T_{s}$ is certainly an overestimate, one can state that Geminga's temperature is incompatible with these cooling models (unless Geminga's age is underestimated, but spin-down ages are usually considered to be overestimates). With pairing of the protons (b) the discrepancy increases because the small $(\sim 25 \%)$ decrease of the specific heat in the photon cooling era is not sufficient to compensate for the significant reduction of the earlier core neutrino emission (during which only the very slow $n n$ bremsstrahlung is unaffected), which gives a high temperature at the beginning of the photon cooling era. When all the core neutrons are paired (c) the reduction of $C_{v}$ is large enough to accomodate the observed temperature. If both neutrons and protons are paired within the whole core (d) $C_{v}$ is cut by a factor of 20 (only the electron and muon contributions are left) and the temperature drop during the photon cooling era is extremely fast; however, heating mechanisms (Shibazaki \& Lamb 1989; Cheng et al. 1992; Umeda et al. 1993) could slow the cooling and keep the temperature compatible with Geminga. These results were at a fixed mass of $1.4 M_{\odot}$, but Figure 3 shows that changing the star mass makes little difference as long as pairing is still assumed throughout the whole core. With realistic density dependent gaps, by varying the star's mass we change the fraction of core baryons paired, and any temperature between the extreme cases of Figures $2 \mathrm{~b}$ and $2 \mathrm{~d}$ could in principle be obtained.

Figure 4 shows three cooling curves with three published calculations of ${ }^{3} P_{2}$ neutron pairing and the EOS WFF(av14) for a $1.4 M_{\odot}$ star. (The Fermi momentum of the neutrons in the center of the star, for comparison with Fig. 1, is $\left.k_{F}(n)=2.58 \mathrm{fm}^{-1}\right)$. The pairing of Takatsuka (1972) has almost no effect, since $T_{c}$ vanishes at low density and most of the core is left unpaired. The two calculations of Hoffberg et al. (1970) and Amundsen \& Ostgaard (1985b) give almost identical results in the photon cooling era, since in both cases the whole core is paired and the internal temperature $T_{i}$ is much lower than $T_{c}$; in both cases, the whole core neutron specific heat has been practically eliminated. In the neutrino cooling era where $T_{i}$ is higher these two cases do differ significantly (their $T_{c}$ differ by an order of magnitude): in the Hoffberg et al. (1970) case, the core neutrino emission is 
practically turned off early on due to the very high $T_{c}$, while in the Amundsen \& Østgaard (1985b) case, the suppression happens much later and is thus less efficient. It is thus not possible to deduce any value for $T_{c}$ from the Geminga data only, except that it must be higher than a few multiples of $10^{8} \mathrm{~K}$, but we can state that pairing must occur within most of the core. Published cooling curves with the PS EOS (Nomoto \& Tsuruta 1986, 1987; Van Riper 1991) also fit the Geminga data, in their superfluid versions where the neutrons are paired in the whole core.

\section{COMMENTS}

\subsection{The Cooling of Low-Mass Neutron Stars}

The surface temperatures we have obtained with the slow neutrino cooling scenario during the photon cooling era when both neutrons and protons are paired within the whole core (Fig. 2 $\mathrm{d}$ ) are much lower than any prediction previously published. This is so because $95 \%$ of the star's specific heat has been suppressed by superfluidity when all core nucleons are paired. This case has to be seriously considered for low-mass neutron stars, where there is little doubt that pairing happens down to the center of the star for both neutrons and protons, and it has some unexpected effects. Consider, e.g. as shown in Figure 5, the case of a heavy star undergoing fast neutrino cooling with suppression from neutron ${ }^{3} P_{2}$ pairing but with a core that still has a substantial amount of unpaired protons. If the critical temperature for neutron pairing is a few multiples of $10^{9} \mathrm{~K}$, during the neutrino cooling era the star will have a lower temperature than a slow neutrino cooling star of low mass, but

later during the photon cooling era it will be much warmer than the lighter (wholly paired) star. Thus fast neutrino cooling does not mean fast cooling forever.

If one adopts the idea that the critical density for the onset of fast neutrino emission is low, then it is quite possible that all neutron stars undergoing slow neutrino cooling, and thus having very low central densities, have both their neutrons and their protons paired within the whole core with high values of $T_{c}$. All neutron stars undergoing slow neutrino cooling would then cool very quickly in the photon cooling era and would become invisible after a couple of hundreds of thousands of years. Consequently, any neutron star older than that, with detectable surface thermal emission, has an unpaired baryonic core component, which provides the star with a sizable specific heat, but has undergone fast neutrino emission suppressed early on by pairing of its other core component(s). 


\section{2. "Standard" Cooling?}

However, there may not even be such a thing as a slow neutrino cooling ("standard" cooling) neutron star since, as mentioned in the introduction, the number of presently proposed channels for fast neutrino emission is so large, and the corresponding critical densities so low in some cases, that it is possible that all neutron stars cool by some fast neutrino process. If this is the case, the thermal evolution of all neutron stars is entirely controlled by superfluidity. The surface temperature from age $\sim 10^{2}$ yr to $\sim 10^{5}$ yr depends on the minimal value of $T_{c}$ for the relevent neutrino emitting baryonic component (nucleon or hyperon) in the core (Page \& Applegate 1992), and the temperature from age $\sim 10^{5}$ yr to $\sim 10^{6}$ yr depends on how much of the core is left unpaired. It is worth mentioning here that observational support for the "standard" model is actually extremely dim, if not nonexistent. It has traditionally been based (Tsuruta 1986) on Einstein observations of the Crab pulsar (Harnden \& Seward 1984) and of the two compact objects detected in the supernova remnants 3C58 (Becker et al. 1982) and RCW 103 (Tuohy et al 1983), all

three having ages $\sim 1000 \mathrm{yr}$ and upper limits on temperature of the order of $2-3 \times 10^{6} \mathrm{~K}$. However, none of these three temperature estimates can be given much credibility for the following reasons: 1) ROSAT has failed to detect the previously seen source in RCW 103 (Becker et al 1993); no matter what this object is, or was, it is not a neutron star cooling according to the slow neutrino emission scenario. 2) The temperature estimate for the 3C58 central source was based on an assumed distance of $8 \mathrm{kpc}$ which was later reduced by a factor of 3 (Green \& Gull 1982): with this new distance, the resulting temperature would be low enough to be marginally inconsistent with the "standard" cooling model. Moreover the age of 3C58 is based on an association with the A.D. 1191 supernova, but Becker et al. (1982) questioned this association, arguing from the low ratio of X-ray to radio luminosities of the remnant that it is probably much older; this would ruin its relevance for comparison with models of early cooling of neutron stars. 3) For the Crab pulsar (the only case of these three whose existence and age are beyond doubt), the temperature estimate is based on an upper limit of the flux between the pulses of the X-ray curve observed by Einstein: the pulsar is undetected at this phase, and thus the temperature can hence be anywhere below the reported upper limit of $2.5 \times 10^{6} \mathrm{~K}$. In none of these three cases was there any spectral evidence that the X-ray emission is thermal emission from the surface of the star, since the Crab and RCW 103 observations were done with the HRI detector, which had no energy resolution, and the count rate from the 3C58 point source in the IPC detector was too low to provide useful spectral information. Moreover, the magnetospheric X-ray emission from such young neutron stars is so strong that there is little hope of detecting thermal radiation from the surface of the star itself (Ögelman 1993). A fourth neutron star young enough to allow us to distinguish between slow and fast neutrino cooling, and in this case 
with good data, is PSR 0833-45 (Vela): comparisons with theoretical models have shown repeatedly that its surface temperature is too low for "standard" cooling and requires a fast cooling agent (Nomoto \& Tsuruta 1986; Shibazaki \& Lamb 1989; Page \& Baron 1990; Van Riper 1991; Page \& Applegate 1992; Umeda et al 1992, 1993). It is therefore a reasonable theoretical a priori to interpret all data within the fast neutrino cooling scenario. Nevertheless, as stated in section 3.1, we still consider both types of neutrino cooling, letting observation be the ultimate judge.

\subsection{PSR 0656+14 and PSR 1055-52}

Since analyses of the ROSAT observations of PSR 0656+14 and PSR 1055-52 have been published recently, we will now briefly comment on these results in light of the preceding remarks. The data are plotted in Figure 6, along with some typical cooling curves.

In the case of PSR 0656+14, when the spectral fit is done with a blackbody, the resulting surface temperature is $9.0 \pm 0.4 \times 10^{5} \mathrm{~K}$, while a nonmagnetic helium atmosphere gives $2.2 \pm 0.2 \times 10^{5} \mathrm{~K}$ (Finley et al. 1992). For a spin-down age of $1.1 \times 10^{5} \mathrm{yr}$ the first value is perfectly compatible with the slow neutrino cooling scenario (without core pairing or with an appropriate proportion of core baryons paired), while the second is in disagreement and needs fast neutrino cooling, unless the star is older than its spin-down age. An analysis with a magnetic hydrogen atmosphere spectrum gives an intermediate value of $6.9_{-0.3}^{+0.5} \times 10^{5} \mathrm{~K}$ (Anderson et al. 1993), which is lower than previously predicted for slow neutrino cooling but slightly higher than our new results in the case of complete pairing of the core: this temperature can easily be accomodated within this model by having almost complete pairing of neutrons and protons in the core, i.e. by slightly increasing the total specific heat compared to the completely paired case. PSR $0656+14$ would have to be much younger than its spin-down age indicates for the magnetic temperature estimate to be incompatible with the slow neutrino cooling scenario.

For PSR 1055-52, spectral fits with a blackbody give a surface temperature of $7.0 \pm 0.6 \times 10^{5} \mathrm{~K}$ (Ögelman \& Finley 1993). Given the spin-down age of $5 \times 10^{5}$ years, Ögelman \& Finley conclude that this temperature is compatible with the slow neutrino cooling models: this is true only for models without pairing in the core or with only protons paired. This temperature is slightly too high compared to slow cooling whith neutron core pairing, but heating may explain the discrepancy, and moreover, fits with non black-body spectra will give lower temperatures and require less heating, if any at all. If both neutrons and protons are paired within the whole core, the theoretical temperature with slow 
neutrino cooling is much lower than the $7 \times 10^{5} \mathrm{~K}$ reported. If we compare this result with those from the heating models of Shibazaki \& Lamb (1989) and Cheng et al (1992) only the maximum heating rates of these models could justify the discrepancy in this case: thus, PSR 1055-52 most probably contains an unpaired component in its core. Being more speculative, if one adopts the idea that the critical density for fast neutrino emission is low and that, consequently, all neutron stars undergoing slow neutrino cooling have their whole core paired and follow the trajectory of Figure $2 \mathrm{~d}$, then this reported temperature of $7 \times 10^{5} \mathrm{~K}$ is incompatible with the slow neutrino cooling scenario unless a very efficient heating mechanism is at work.

\section{CONCLUSIONS}

We have compared the recent temperature measurement of the Geminga neutron star with cooling models and found that, since this star is old enough to be in the photon cooling era, both fast and slow neutrino emission mechanisms can explain its temperature. One therefore cannot draw any conclusion about neutrino emission from dense nuclear matter using this observation alone. However, a crucial feature in both types of models is that they need nucleon pairing in most, if not all, of the core. If no pairing is assumed in the core, then the predicted temperature is either too high (slow neutrino cooling) or too low (fast neutrino cooling) when compared to the observed temperature of Geminga.

With fast neutrino cooling, nucleon pairing is needed to stop the early cooling which, without this, would produce a star with a temperature much lower than what is observed. If the fast neutrino emission is from hyperonic processes it is possible that the suppression we

observe is due to hyperon superfluidity. It is not possible to distinguish between the various fast processes, however; the theoretical uncertainty about $T_{c}$ allows us to accomodate very different neutrino emission rates. Moreover, within a given fast cooling scenario, the observational uncertainty also allows very different values of $T_{c}$. However, since fast neutrino emission occurs down to the very center of the core, to be compatible with the Geminga observation, these scenarios need pairing up to the highest density reached in this object, with pairing critical temperatures higher than $10^{9} \mathrm{~K}$.

Within the slow neutrino cooling model (the "standard" model), superfluidity is also needed, but for a different reason. The observed temperature is below what the simple model without pairing predicts, but since this star is cooling by photon emission, we can accelerate the cooling at this time by decreasing the specific heat through pairing. The theoretical curves, in the photon cooling era, are very insensitive to the high-density EOS 
or the star mass, the only determining factor being how much of the core specific heat has been eliminated by pairing. If we accept an age of $3 \times 10^{5} \mathrm{yr}$ and a temperature of $5 \times 10^{5}$ $\mathrm{K}$, then the specific heat must have been reduced to about $25 \%$ of its normal value. This can be obtained either by pairing of the neutrons in the whole core or by a combination of neutron and proton pairing, but even in this case most of the neutrons must be paired. If both neutrons and protons are paired in the whole core, photon cooling becomes so efficient that a substantial amount of heating is needed, but several possible mechanisms have been proposed and may be able to provide sufficient heating.

The above discussion shows that, in order to distinguish clearly between the fast and slow neutrino cooling scenarios, we need observations of neutron stars younger than $5 \times 10^{4}$ $\mathrm{yr}$, for which the slow cooling scenario predicts temperatures higher than $0.9-1.1 \times 10^{6} \mathrm{~K}$, depending on the exact age; at later times, both scenarios can accomodate most observable temperatures depending on the amount of pairing assumed. Geminga is old enough that the effect of the early neutrino cooling has been washed out. However, our analysis showed that this star does tell us -independently of its earlier neutrino cooling history- that most, if not all, of its core is paired. PSR $0656+14$ is at the limit at which we can still distinguish the effect of fast neutino cooling, but the present uncertainty about its temperature precludes drawing any conclusion. PSR 1055-52 can be also interpreted within both types of neutrino scenarios, but its core must contain an unpaired component whose specific heat keeps the star warm despite its age. If one accepts the fast neutrino cooling scenario as universal (a reasonable theoretical a priori), then these three objects show evidence of baryon pairing down to their very center, needed for suppression of early neutrino emission, but none of them requires this scenario.

I am grateful to T. Ainsworth, J. H. Applegate, J. P. Halpern, M. Prakash, and M. Ruderman for discussions. This work was supported by HEA-NASA grant NAGW 3075 and, in its early phases, by a fellowship from the Swiss National Science Foundation. This work is contribution number 544 of the Columbia Astrophysics Laboratory. 


\begin{tabular}{lcc}
\hline \multicolumn{1}{c}{ Process Name } & Process & $\begin{array}{c}\text { Emissivity } Q_{\nu} \\
\left(\mathrm{erg} / \mathrm{sec} / \mathrm{cm}^{3}\right)\end{array}$ \\
\hline a) Modified URCA & $\left\{\begin{array}{l}n+n^{\prime} \rightarrow n^{\prime}+p+e^{-}+\overline{\nu_{e}} \\
n^{\prime}+p+e^{-} \rightarrow n^{\prime}+n+\nu_{e}\end{array}\right.$ & $\sim 10^{20} \cdot T_{9}^{8}$ \\
b) K-condensate & $\left\{\begin{array}{l}n+K^{-} \rightarrow n+e^{-}+\overline{\nu_{e}} \\
n+e^{-} \rightarrow n+K^{-}+\nu_{e}\end{array}\right.$ & $\sim 10^{24} \cdot T_{9}^{6}$ \\
c) $\pi$ - condensate & $\left\{\begin{array}{l}n+\pi^{-} \rightarrow n+e^{-}+\overline{\nu_{e}} \\
n+e^{-} \rightarrow n+\pi^{-}+\nu_{e}\end{array}\right.$ & $\sim 10^{26} \cdot T_{9}^{6}$ \\
d) Direct URCA & $\left\{\begin{array}{l}n \rightarrow p+e^{-}+\overline{\nu_{e}} \\
p+e^{-} \rightarrow n+\nu_{e}\end{array}\right.$ & $\sim 10^{27} \cdot T_{9}^{6}$ \\
e) Quark URCA & $\left\{\begin{array}{l}d \rightarrow u+e^{-}+\overline{\nu_{e}} \\
u+e^{-} \rightarrow d+\nu_{e}\end{array}\right.$ & $\sim 10^{26} \alpha_{c} T_{9}^{6}$
\end{tabular}

Table 1: Some core neutrino emission processes and their emissivities. The emissivities are from : a) Friman \& Maxwell 1979, b) Brown et al 1988, c) Maxwell et al 1977, d) Lattimer et al 1991 and e) Iwamoto 1980. $T_{9}$ is the temperature in units of $10^{9}$ kelvins. 


\begin{tabular}{ccccccc}
\hline EOS & \multicolumn{3}{c}{ Maximum-Mass Star } & \multicolumn{3}{c}{$1.4 M_{\odot}$ Star } \\
. & $M\left(M_{\odot}\right)$ & $\rho_{c}\left(\mathrm{fm}^{-3}\right)$ & $x_{p}(\%)$ & $R(\mathrm{~km})$ & $\rho_{c}\left(\mathrm{fm}^{-3}\right)$ & $x_{p}(\%)$ \\
\hline FP & 1.79 & 1.18 & $\sim 0$ & 10.85 & 0.69 & 2.0 \\
WFF(av14) & 2.10 & 1.25 & 4.8 & 10.60 & 0.64 & 9.6 \\
MPA & 2.44 & 0.89 & 19.0 & 12.45 & 0.41 & 9.0 \\
PAL32 & 1.68 & 1.51 & 15.2 & 11.02 & 0.74 & 11.4 \\
PAL33 & 1.90 & 1.24 & 14.0 & 11.91 & 0.54 & 9.7 \\
\hline
\end{tabular}

Table 2: Some properties of the EOS's used for slow neutrino cooling. Columns two to four list the mass, central density and central proton fraction of a maximum mass star. Columns five to seven give properties of a $1.4 \mathrm{M}_{\odot}$ star : radius, central density and central proton fraction. (The PAL EOSs are labeled PALij, i,j=1,2,3, where i refers to the symmetry energy function and $\mathrm{j}$ to the compression modulus) 


\begin{tabular}{cccccc}
\hline EOS & \multicolumn{3}{c}{ Core Components } & \multicolumn{2}{c}{ Crust Components } \\
& $\mathrm{n}$ & $\mathrm{p}$ & $\mathrm{e}$ & $\mathrm{n}$ & $\mathrm{e}$ \\
\hline FP & 9.89 & 2.89 & 0.50 & 0.97 & 0.025 \\
WFF(av14) & 9.31 & 2.81 & 0.51 & 0.72 & 0.018 \\
MPA & 11.90 & 3.90 & 0.67 & 1.60 & 0.044 \\
PAL32 & 9.66 & 3.41 & 0.68 & 1.16 & 0.031 \\
PAL33 & 10.96 & 3.72 & 0.68 & 1.42 & 0.037 \\
\hline
\end{tabular}

Table 3: Normal specific heat, at $T=10^{9} \mathrm{~K}$, of neutrons, protons and electrons in the core and crust of a $1.4 M_{\odot}$ neutron star built with the five EOS's used for slow neutrino cooling. (Units are $10^{38}$ ergs $K^{-1}$, i.e. $C_{v}(T)=($ Table - Entry $) \times\left(T / 10^{9} K\right) \times 10^{38}$ ergs $\left.K^{-1}\right)$. 


\section{REFERENCES}

Ainsworth, T. L. 1992, private communication

Ainsworth, T. L., Wambach, J. \& Pines, D. 1989, Phys. Lett., B222, 173

Amundsen, L. \& Østgaard, E. 1985a, Nucl. Phys., A437, 487

Amundsen, L. \& Østgaard, E. 1985b, Nucl. Phys., A442, 163

Anderson, S. B., Córdova, F. A., Pavlov, G. G., Robinson, C. R. \& Thompson, Jr, R. J. 1993, ApJ, 414, 867

Bailin, D. \& Love, A. 1984, Phys. Rep., 107, 325

Baym, G., Pethick, C. J. \& Sutherland, P. 1971, ApJ, 170,299

Becker, R. H., Helfand, D. J. \& Szymkowiak, A. E. 1982, ApJ, 255, 557

Becker, W., Trümper, J., Hasinger, G. \& Aschenbach B. 1993, in Isolated Pulsars, ed. K. A. Van Riper, R. Epstein \& C. Ho (Cambridge: Cambridge Univ. Press) 116

Bignami, G. F. \& Caraveo, P. A. 1992, Nature, 357, 287

Brown, G. E., Kubodera, K., Page, D. \& Pizzochero, P. 1988, Phys. Rev., D37, 2042

Chao, N.-C., Clark, J. W. \& Yang, C.-H. 1972, Nucl. Phys., A179, 320

Cheng, K. S., Chau, W. Y., Zhang, J. L. \& Chau, H. F. 1992, ApJ, 396, 135

Finley, J. P., Ögelman, H. \& Kiziloğlu, Ü 1992, ApJ, 394, L21

Friedman, B. \& Pandharipande, V. R. 1981, Nucl. Phys., A361, 502

Friman, B. L. \& Maxwell, O. V. 1979, ApJ, 232, 541

Gehrels, N. \& Chen, W. 1993, Nature, 361, 706

Glendenning, N. K. 1985, ApJ, 293, 470

Gnedin, O. Y. \& Yakovlev, D. G. 1993, Astron. Lett., 19, 104

Green, D. A. \& Gull, S. F. 1982, Nature, 299, 606

Gudmundsson, E. H., Pethick, C. J. \& Epstein, R. I. 1982, ApJ, 259, L19

Gudmundsson, E. H., Pethick, C. J. \& Epstein, R. I. 1983, ApJ, 272, 286

Halpern, J. P. and Holt, S. S. 1992, Nature, 357, 222

Halpern, J. P. \& Ruderman, M. 1993, ApJ, 415, 286

Harnden, F. R. \& Seward, F. D. 1984, ApJ, 283, 279

Hernquist, L. 1985, MNRAS, 213, 313 
Hoffberg, M., Glassgold, A. E., Richardson, R. W. \& Ruderman, M. 1970, Phys. Rev. Lett., 24, 775

Iwamoto, N. 1980, Phys. Rev. Lett.,44, 1637

Jackson, A. D., Krotscheck, E., Meltzer, D. E. \& Smith, R. A. 1982, Nucl. Phys., A386, 125

Lattimer, J. M., Pethick, C. J., Prakash, M. \& Haensel, P. 1991, Phys. Rev. Lett., 66, 2701

Levenfish, K. P. \& Yakovlev, D. G. 1993, in Strongly Coupled Plasma Physics, ed. H. M. Van Horn \& S. Ichimaru (Rochester: University of Rochester Press)

Lyne, A. G. \& Graham-Smith, F. 1990, Pulsar Astronomy, (Cambridge: Cambridge University Press)

Maxwell, O. V. 1987, ApJ, 316, 691

Maxwell, O. V., Brown, G. E., Campbell, D. K., Dashen, R. F. \& Manassah, J. T. 1977, ApJ, 216, 77

Michel, F. C. 1991, Theory of Neutron Star Magnetospheres, (Chicago: Univ. Chicago Press)

Miller, M. C. 1992, MNRAS, 255, 129

Müther, H., Prakash, M. \& Ainsworth, T. L. 1987, Phys. Lett., B199, 469

Niskanen, J. A. \& Sauls, J. A. 1981, preprint

Nomoto, K. \& Tsuruta, S. 1986, ApJ, 305, L19

Nomoto, K. \& Tsuruta, S. 1987, ApJ, 312, 711

Ögelman, H., 1991, in Neutron Stars: Theory and Observation, ed. J. Ventura \& D. Pines (Dordrecht: Kluwer Academic Publisher) 87

Ögelman, H., 1993, in Isolated Pulsars, ed. K. A. Van Riper, R. Epstein \& C. Ho (Cambridge : Cambridge University Press) 96

Ögelman H. \& Finley J. P. 1993, ApJ, 413, L31

Page, D. 1989, Ph.D. thesis, SUNY Stony Brook

Page, D. 1992, in Proc. 1st Symp. Nuclear Physics in the Universe, ed. M. R. Strayer \& M. W. Guidry (Bristol: Adam Hilger) 151

Page, D. 1994, in preparation

Page, D. \& Applegate, J. H. 1992, ApJ, 394, L17

Page, D. \& Baron, E. 1990, ApJ, 354, L17; Erratum in ApJ, 382, L111

Pandharipande, V. R. 1971, Nucl. Phys., A178, 123 
Pandharipande, V. R., Pines, D. \& Smith, R. A. 1976, ApJ, 208, 550

Pethick, C. J. 1992, Rev. Mod. Phys., 64, 1133

Prakash, M. 1993, Phys. Rep., in press

Prakash, M., Ainsworth, T. L. \& Lattimer, J. M. 1988, Phys. Rev. Lett., 61, 2518

Prakash, M., Prakash, M., Lattimer, J. \& Pethick, C. J. 1992, ApJ., 390, L77

Romani, R. W. 1987, ApJ, 313, 718

Schaaf, M. E. 1990a, A\&A, 227, 61

Schaaf, M. E. 1990b, A\&A, 235, 499

Shibanov, Y. A., Zavlin, V. E., Pavlov, G. G. \& Ventura, J. 1992, A\&A, 266, 313

Shibazaki, N. \& Lamb, F. K. 1989, ApJ, 346, 808

Takatsuka, T. 1972, Prog. Theor. Phys., 48, 1517

Takatsuka, T. 1973, Prog. Theor. Phys., 50, 1754

Takatsuka, T. \& Tamagaki, R. 1982, Prog. Theor. Phys., 67, 1649

Takatsuka, T., Tamiya, K., Tatsumi, T. \& Tamagaki, R. 1978, Prog. Theor. Phys., 59, 1933

Tamagaki, R. 1992, Physica, B178, 13

Tsuruta, S. 1986, Comments Astrophys, 11,151

Tuohy, I. R., Garmire, G. P., Manchester, R. N. \& Dopita, M. A. 1983, ApJ, 268, 778

Umeda, H., Nomoto, K., Tsuruta, S., Muto, T. \& Tatsumi, T. 1992, in The Structure and Evolution of Neutron Stars, ed. D. Pines, R. Tamagaki \& S. Tsuruta (New York: Addison-Wesley) 406

Umeda, H., Shibazaki, N., Nomoto, K. \& Tsuruta, S. 1993, ApJ, 408, 186

Van Riper, K. A. 1988, ApJ, 329, 339

Van Riper, K. A. 1991, ApJS, 75, 449

Wambach, J., Ainsworth, T. L. \& Pines, D. 1991, in Neutron Stars : Theory and Observation, ed. J. Ventura \& D. Pines (Dordrecht: Kluwer) 37

Wiringa, R. B., Fiks, V. \& Fabrocini, A. 1988, Phys. Rev., C38, 1010

This preprint was prepared with the AAS LATEX macros v3.0. 
Fig. 1.- a) Proton ${ }^{1} S_{0}$ pairing critical temperatures. CCY-Chao, Clark \& Yang (1972), T73-Takatsuka (1973), NS-Niskanen \& Sauls (1981), AO-Amundsen \& Østgaard (1985a), WAP-Wambach et al. (1991).

b) Neutron ${ }^{3} P_{2}$ pairing critical temperatures. HGRR-Hoffgerg et al. (1970), T72-Takatsuka (1972), AO-Amundsen \& Østgaard (1985b). The two dashed curves show the results of T72 and $\mathrm{AO}$ when the neutron effective mass is fixed at the free mass value.

In abscissa is the Fermi wave number $k_{F}$, related to the particle number density $n_{i}$ by $k_{F}\left(n_{i}\right)=\left(3 \pi^{2} n\right)^{1 / 3}=1.68\left(n_{i} / n_{0}\right)^{1 / 3} \mathrm{fm}^{-1}$ where $n_{0}=0.16 \mathrm{fm}^{-3}$.

Fig. 2.- Cooling by the modified Urca process: effect of the specific heat suppression by nucleon pairing. The various curves correspond to the five EOSs we use: FP (continuous), WFF(av14) (dashed), MPA (dotted), PAL32 (dash-dotted) and PAL33 (dash-triple-dotted). $1.4 M_{\odot}$ star.

a) No core pairing at all.

b) Protons paired with a density-independent $T_{c}=2 \times 10^{9} \mathrm{~K}$.

c) Core neutrons paired with a density-independent $T_{c}=2 \times 10^{9} \mathrm{~K}$.

d) Protons and core neutrons paired with a density-independent $T_{c}=2 \times 10^{9} \mathrm{~K}$. Crust neutrons are paired according to Ainsworth et al. (1989). The temperature in ordinate is the effective temperature "at infinity," i.e., redshifted. The cross shows Geminga's temperature and age.

Fig. 3.- Cooling by the modified Urca process: effect of the star mass. EOS WFF(av14) and star mass of $1.2 M_{\odot}$ (dash-dotted), $1.4 M_{\odot}$ (continuous), $1.6 M_{\odot}$ (dash-triple dotted) and $1.8 M_{\odot}$ (dashed) with the same pairings as in Fig. 2. Crust neutrons are paired according to Ainsworth et al. (1989). The temperature in ordinate is the effective temperature "at infinity," i.e., redshifted. The cross shows Geminga's temperature and age.

Fig. 4.- Cooling by the modified Urca process: density dependent $T_{c}$. EOS WFF(av14) and star mass $1.4 M_{\odot}$. The continuous curve has no core neutron pairing, the other three curves have core neutron pairing as labeled (see Figure 1b). Core protons are not paired. Crust neutrons are paired according to Ainsworth et al. (1989). The temperature in ordinate is the effective temperature "at infinity," i.e., redshifted. The cross shows Geminga's temperature and age. 
Fig. 5.- Fast neutrino cooling is not fast cooling forever: the curves show the cooling history of two neutron stars of very different masses built with the same EOS, WFF(av14), and the same pairings (HGRR for core neutrons, CCY-PSi) for core protons, as labeled in Figure 1, and Ainsworth et al. 1989 for crust neutrons). With this choice of pairings, neutrons are paired in the whole core for both stars, as are protons in the lighter star while the heavier star has a central region of unpaired protons. We have assumed that a kaon condensate develops above a density of $10^{15} \mathrm{gm} / \mathrm{cm}^{3}$ such that the $1.6 M_{\odot}$ star has a kaon "pit" of $0.56 M_{\odot}$ while the $1.0 M_{\odot}$ star undergoes "standard" cooling. During the neutrino cooling era $\left(30 \mathrm{yr}<\right.$ age $\left.<3 \times 10^{4} \mathrm{yr}\right)$ the lighter star is warmer because of its low neutrino emission, while during the photon cooling era the heavier star is warmer because of its larger specific heat provided by its unpaired protons.

Fig. 6. - Comparison of the estimated surface temperatures of PSR 0656+14 and PSR 1055-52 with theoretical curves of slow neutrino cooling. EOS WFF(av14) and star mass $1.4 M_{\odot}$, no pairing (continuous), protons paired (dotted), neutrons paired (dash-dotted), neutrons and protons paired (dash-triple-dotted). The three temperatures for PSR 0656+14 correspond to blackbody, magnetic hydrogen, and nonmagnetic helium atmospheres as indicated, while the PSR 1055-52 temperature is from a blackbody fit. The age ranges correspond to braking indices from 2 to 4 , as we used for Geminga, the upper values probably being overestimates. 


\section{Author Address}

Dany Page: Instituto de Astronomía, U.N.A.M., Apdo postal 70-264, 04510 MEXICO D.F.

E-mail : PAGE@ASTROSCU.UNAM.MX 
Figure 1
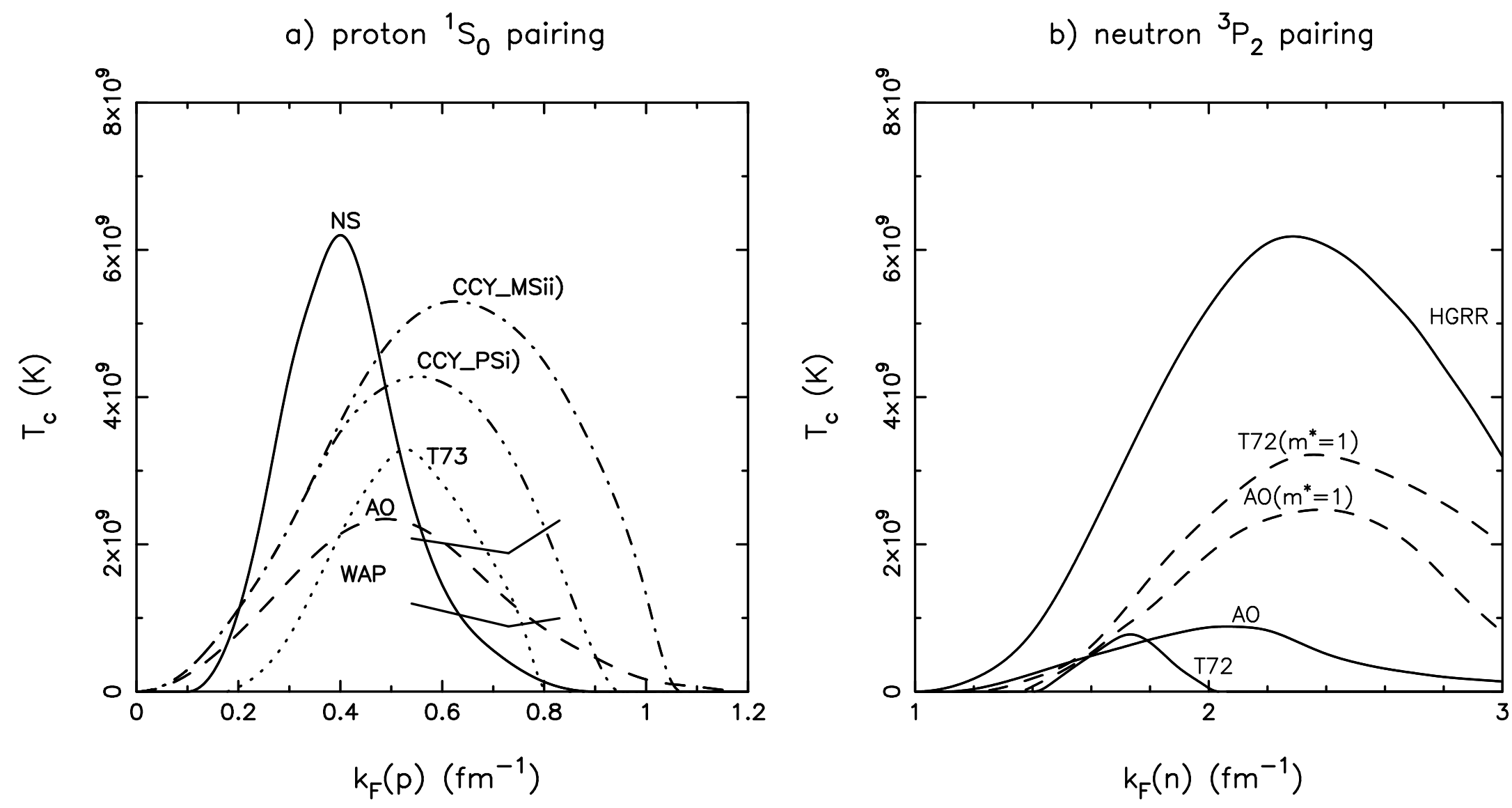
Figure 2
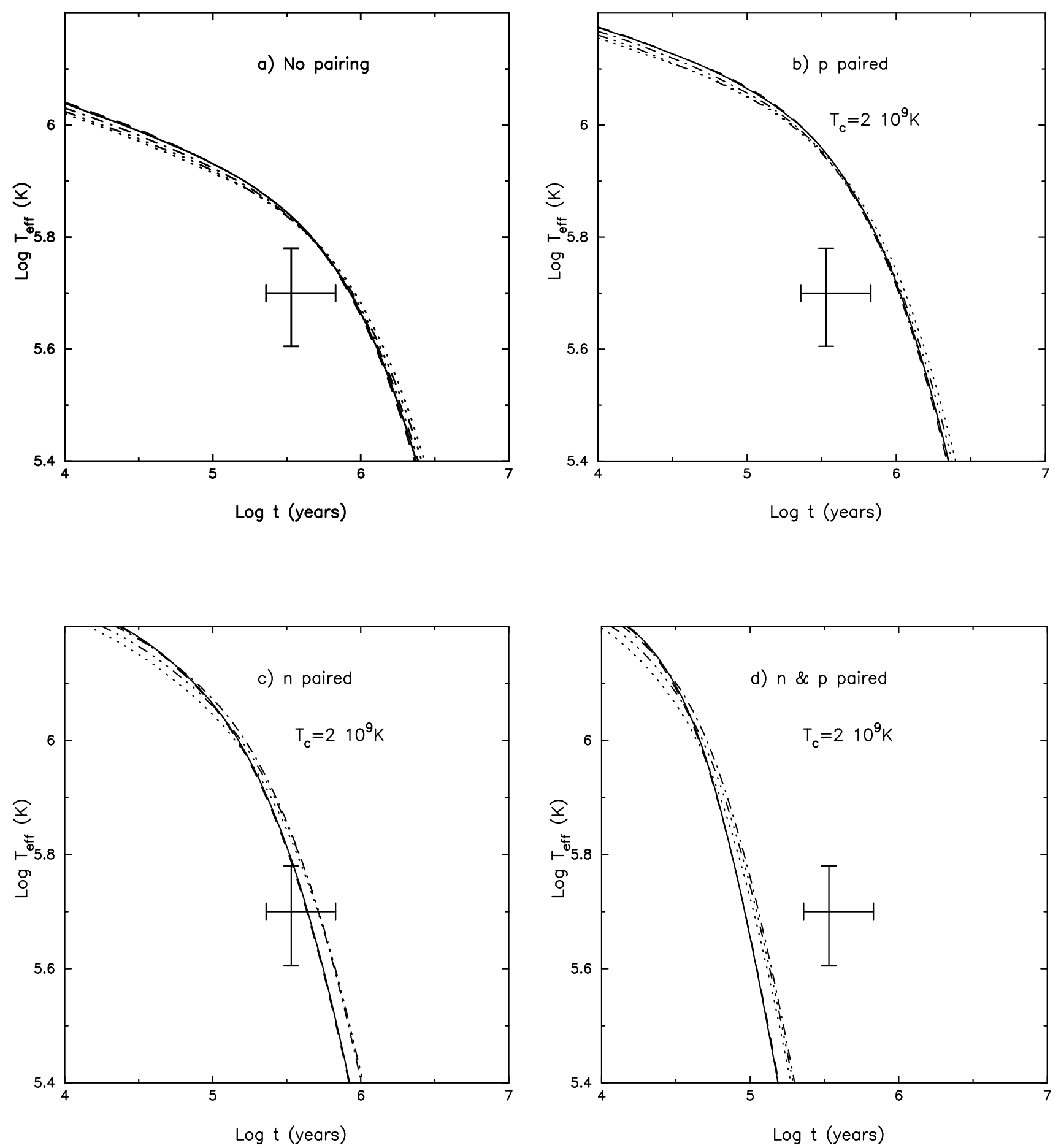
Figure 3

WFF (av14) Mass vs. Pairing

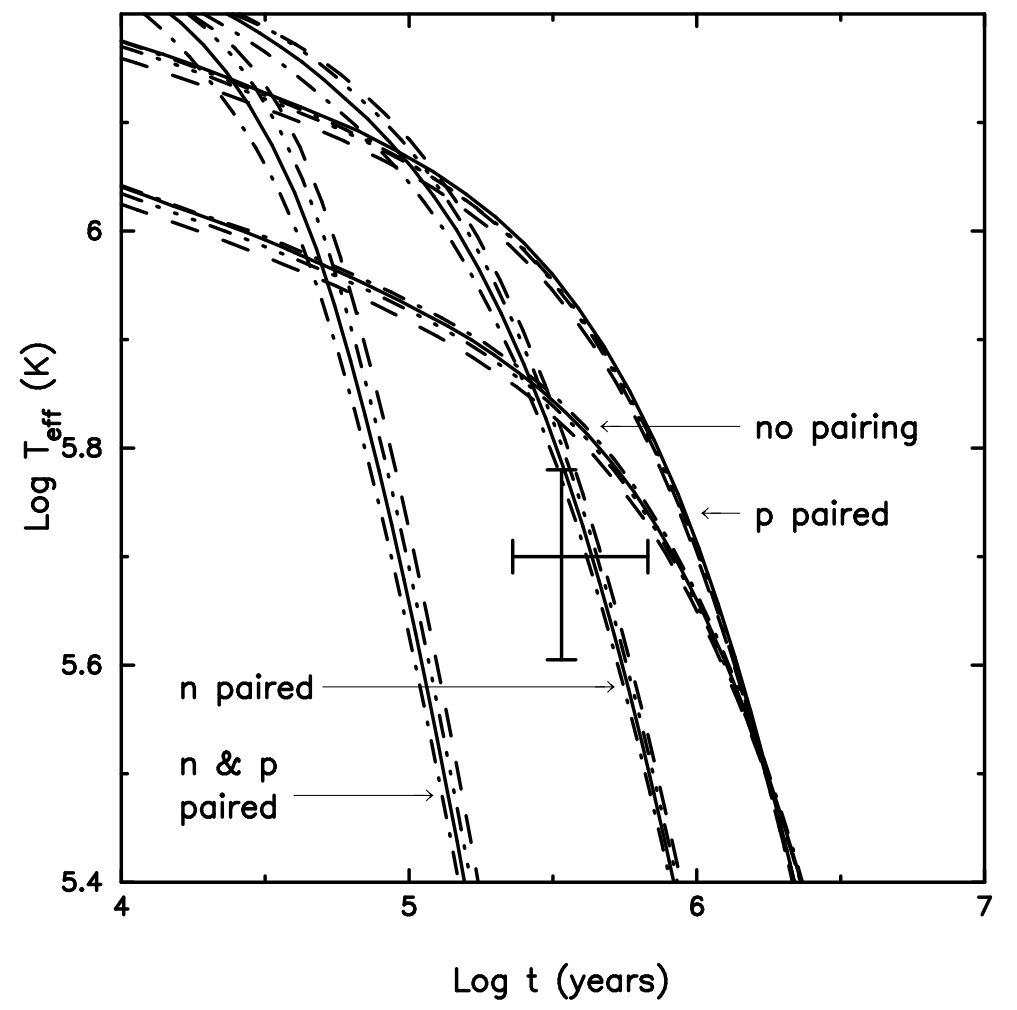

Figure 4

WFF (av14) : various neutron pairing

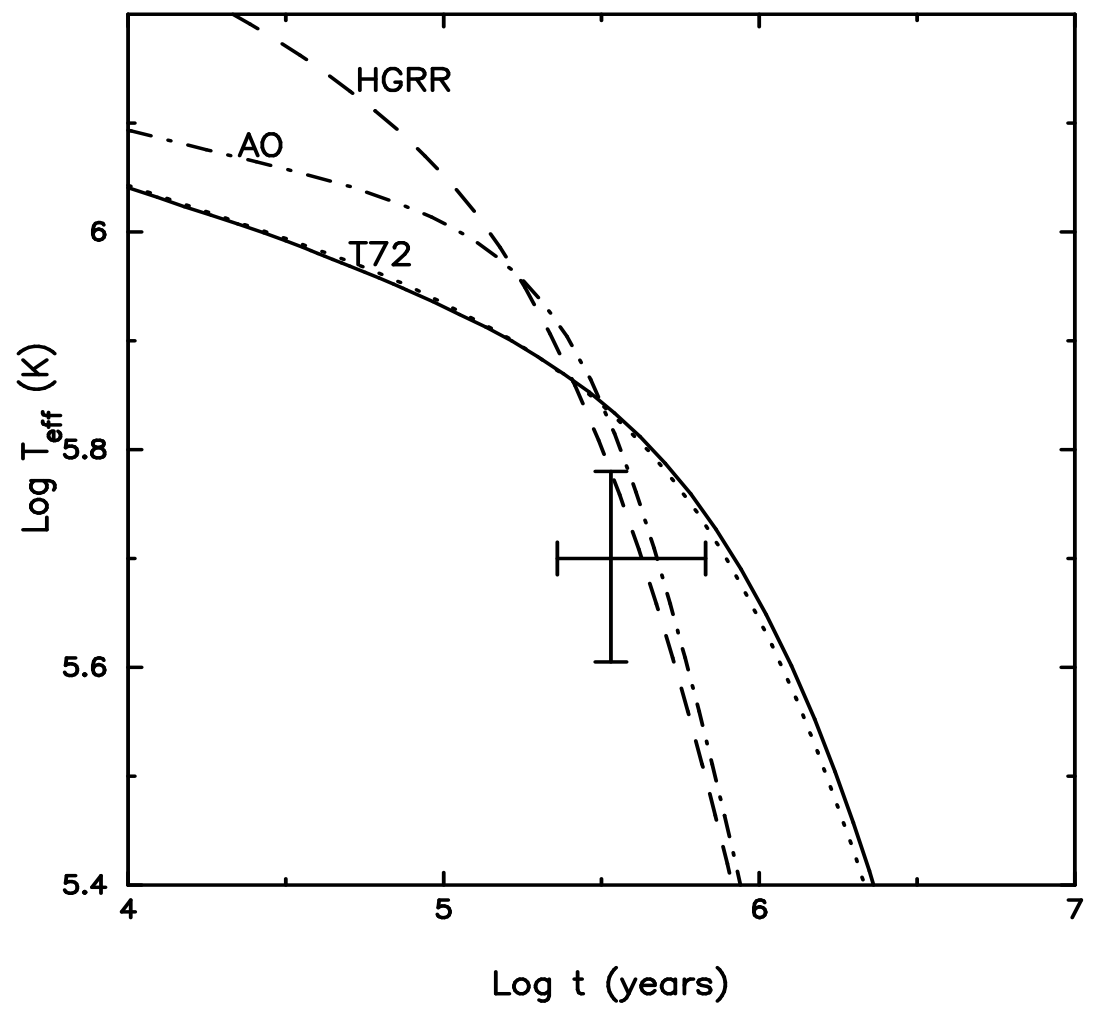


Figure 5

Cooling with/without kaons and core pairing

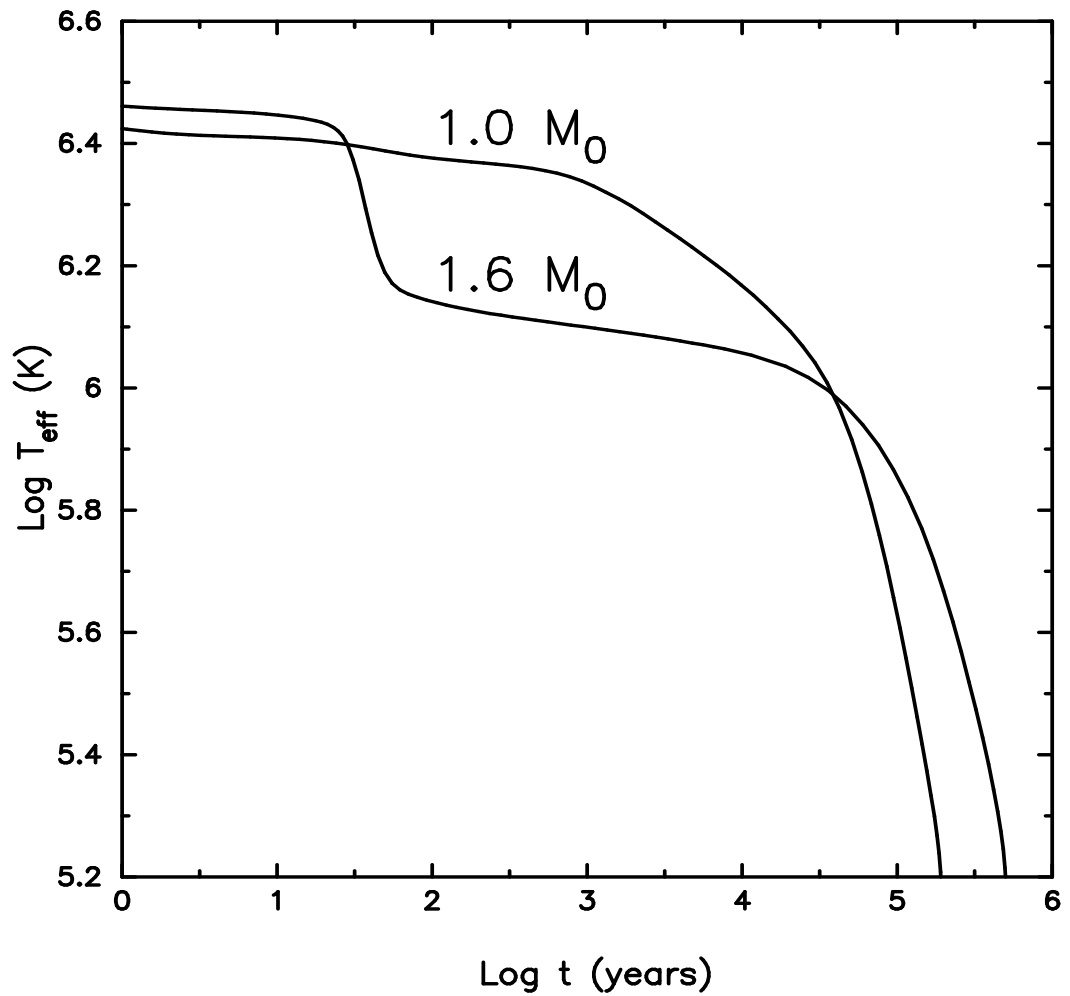

Figure 6

PSR 0656+14 and PSR 1055-52

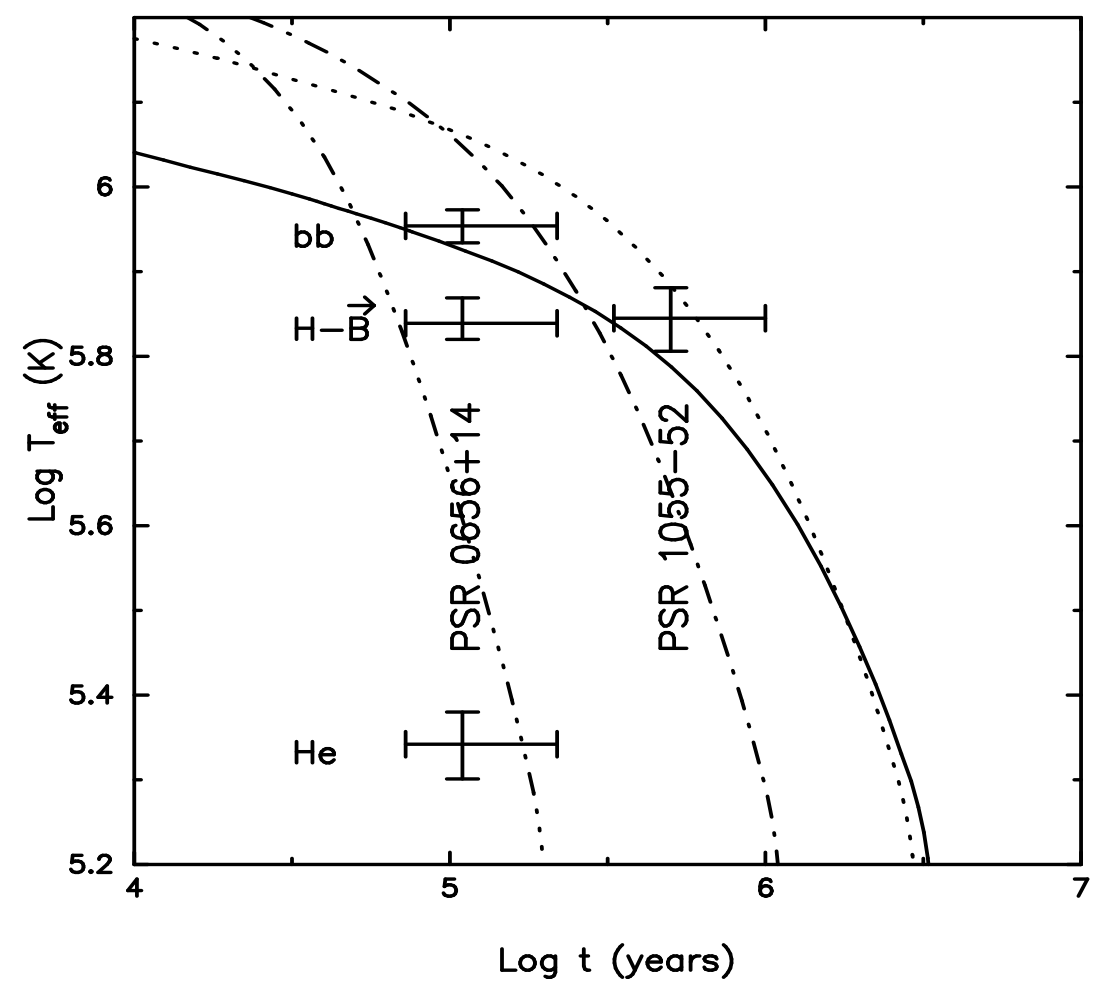

